\title{
Video Article \\ Discrimination and Characterization of Heterocellular Populations Using Quantitative Imaging Techniques
}

\author{
Colleen M. Garvey ${ }^{1}$, Torin A. Gerhart ${ }^{1}$, Shannon M. Mumenthaler ${ }^{1}$ \\ ${ }^{1}$ Lawrence J. Ellison Institute for Transformative Medicine, University of Southern California (USC)
}

Correspondence to: Shannon M. Mumenthaler at smumenth@usc.edu

URL: https://www.jove.com/video/55844

DOI: doi: $10.3791 / 55844$

Keywords: Cellular Biology, Issue 124, quantitative microscopy-based imaging, heterocellular co-cultures, cancer-associated fibroblasts, multiplexed assays, population dynamics

Date Published: 6/30/2017

Citation: Garvey, C.M., Gerhart, T.A., Mumenthaler, S.M. Discrimination and Characterization of Heterocellular Populations Using Quantitative Imaging Techniques. J. Vis. Exp. (124), e55844, doi:10.3791/55844 (2017).

\section{Abstract}

Cellular processes are complex and result from the interplay between multiple cell types and their environment. Existing cell biology techniques often do not allow for accurate interpretation of this interplay. Using a quantitative imaging-based approach, we present a high-content protocol for characterizing the dynamic phenotypic responses (i.e. morphology changes, proliferation, apoptosis) of heterogeneous cell populations to changes in environmental stimuli. We highlight our ability to distinguish between cell types based upon either fluorescence intensity or inherent morphology features depending on the application. This platform allows for a more comprehensive characterization of subpopulation response to perturbation while utilizing shorter time, smaller amounts of reagents, and lower likelihood of error than traditional cell biology assays. However, in some cases, cell populations may be difficult to identify and quantitate based on complex cellular features and will require additional troubleshooting; we highlight some of these circumstances in the protocol. We demonstrate this application using response to drug in a cancer model; however, it can easily be applied more broadly to other physiological processes. This protocol allows one to identify subpopulations within a co-culture system and characterize the particular response of each to external stimuli.

\section{Video Link}

The video component of this article can be found at https://www.jove.com/video/55844/

\section{Introduction}

Cell-based assays have been a workhorse in basic research and drug development settings. However, the limitations of these standard assays have become increasingly apparent with the discordance between in vitro and clinical data and the failure of most drugs to receive FDA approval. Here, we present a novel method for utilizing quantitative imaging to simultaneously analyze heterocellular phenotypes in response to relevant, co-occurring environmental stimuli.

Traditional cell-based assays that are used to measure cell viability include: trypan blue exclusion assays, MTT/MTS, and Annexin V-FITC flow cytometry staining. Trypan blue exclusion assays, while simple and inexpensive, require a large number of cells, are time consuming, and are often influenced by user bias ${ }^{1}$. MTT and MTS assays indirectly measure cell viability through measurements of mitochondrial metabolic rate. However, the metabolic activity of cells can be affected by different culture conditions (such as media or oxygen concentration), which leads to inaccurate results and prevents standardization across cell types and conditions $s^{2,3}$. Another major disadvantage of these techniques is their inability to distinguish between multiple cell types - most biological systems are heterocellular. While flow cytometry methods have the ability to distinguish between multiple cell populations, cell labels are required, dynamic sampling is challenging, and when using adherent cells, this application becomes time-consuming and error prone.

Other important cellular phenotypes, including morphological changes, occur in response to environmental stimuli but are not captured by traditional cell-based assays. Profiling cell states through morphological characterization and mapping similarities across samples is a powerful, unbiased tool with the ability to provide novel insights into many aspects of basic and translational research, including basic cell biology and drug discovery ${ }^{4}$. Furthermore, tumor cell morphology has been shown to correlate with tumor subtypes ${ }^{5}$ and aggressiveness ${ }^{6}$. Hence, it is of great interest to study these cellular features and how they relate to specific environmental perturbations. Additionally, one can use differences in morphological features to discriminate between subpopulations in co-culture systems. Fluorescently labeling cells has downfalls (i.e. altering inherent cell properties, time-consuming) and therefore additional methods to classify cell types are advantageous.

Microscopy-based imaging is an alternative method for profiling cellular phenotypes in a multiplexed, quantitative, and robust manner. In this manuscript, we apply our quantitative imaging pipeline to highlight the evolutionary dynamics of heterogeneous cell populations within a tumor. We focus on the interaction between Non-Small Cell Lung Cancer (NSCLC) cells and Cancer-Associated Fibroblasts (CAFs), the most prevalent stromal cell type found in tumors. CAFs have been implicated in tumor initiation, progression, and therapeutic response; therefore, performing phenotypic assays on tumor cells in the absence of CAFs can be misleading ${ }^{7,8,9}$. Specifically, we evaluated effects of CAFs on tumor cells in response to erlotinib, a small molecule targeting the Epidermal Growth Factor Receptor (EGFR) that is often used in clinical treatment of 
NSCLC. We utilized a high-content screening platform and its accompanying image analysis software for evaluation; however, in an attempt to make this methodology accessible to other researchers we have also developed a comparable downstream protocol using the open-source software: CellProfiler ${ }^{10}$ and CellProfiler Analys ${ }^{11}$. Most image-based high-content screening assays are analyzed with commercialized software specific to a given instrument model. Results are difficult to replicate in other labs with different software because the underlying algorithms are often proprietary. Using this image-based pipeline, cell proliferation, death, and morphology of each subpopulation of a heterocellular culture in response to drug treatment using both fluorescence- and morphology-based classification were measured. The following protocol provides a robust methodology for probing complex cellular processes.

\section{Protocol}

\section{Cell Culture}

NOTE: Here phenotypic responses of NSCLC cells (H3255) to the EGFR-targeting agent, erlotinib, when co-cultured with lung fibroblasts (CCD-19Lu GFP) were investigated. For the same data set, fluorescence-based and morphology-based classification of the two cell populations (H3255 and CCD-19Lu GFP) to exemplify their concordance was performed. However, only one classification method needs to be used and should be chosen based upon the application.

1. Prepare $500 \mathrm{~mL}$ of RPMI-1640 supplemented with $10 \%$ heat-inactivated FBS and $1 \%$ penicillin/streptomycin. NOTE: The growth medium and supplements can be replaced as required for other cell types.

2. Culture cells in $10 \mathrm{~cm}^{3}$ plates as pure populations in $5 \% \mathrm{CO}_{2}$ at $37^{\circ} \mathrm{C}$ and passage the at appropriate ratio every 3-4 days

\section{Preparation of Cells}

1. To prepare a cell suspension, remove cell media and wash cells with $5 \mathrm{~mL} 1 \mathrm{X}$ Phosphate-Buffered Saline (PBS).

2. Aspirate off the PBS, add $1 \mathrm{~mL}$ of $0.05 \%$ trypsin warmed to $37^{\circ} \mathrm{C}$ and incubate for $5 \mathrm{~min}$ (or until cells are no longer adhered to plate) at 37 ${ }^{\circ} \mathrm{C}$.

3. To neutralize trypsin, add $4 \mathrm{~mL}$ of RPMI to $\mathrm{H} 3255$ and CCD-19Lu GFP plates and pipet the cell solutions into $15 \mathrm{~mL}$ conical tubes.

4. Centrifuge cells at $150 \times \mathrm{g}$ for $5 \mathrm{~min}$ at RT.

5. Aspirate off the supernatant and resuspend cell pellets in $10 \mathrm{~mL}$ of RPMI media in conical tubes to prepare for seeding

\section{Cell Plating}

1. Count the cells to standardize seeding.

1. Invert tubes to mix cells in solution. Pipette $10 \mu \mathrm{L}$ of each cell suspension into a microcentrifuge tube and add $10 \mu \mathrm{L}$ trypan blue.

2. Pipette $10 \mu \mathrm{L}$ of this solution into a cell counting slide and insert into automated cell counter. NOTE: Other methods of cell counting (i.e. hemocytometer) may be used.

3. Repeat the cell count at least three times, or until obtained counts are consistent.

2. Seed cells onto a multi-well plate

NOTE: The number of plates to seed is dependent on the number of time points that will be imaged. Alternatively, one plate can be reimaged over time if the cells are transduced with histone-2B-GFP (or other stable lentiviral nuclear stains that provide adequate nuclear segmentation).

1. Seed a total of 1,500 cells/well in $100 \mu \mathrm{L}$ of RPMI. Prepare three cell suspensions to plate three cell populations: H3255, CCD-19Lu GFP, and 50\% H3255 + 50\% CCD-19Lu GFP. Perform each in triplicate with identical plate setups for each time point. NOTE: Initial seeding densities should be optimized for each cell line and plate size.

2. Seed cells in $3 \times 96$-well plates using a multichannel pipette. Incubate cells $\mathrm{O} / \mathrm{N}$ at $37^{\circ} \mathrm{C}, 5 \% \mathrm{CO}_{2}$.

\section{Drug Dosing}

1. Add DMSO to erlotinib powder to make a final erlotinib stock solution of $10 \mathrm{mM}$ concentration. NOTE: The drug can be substituted as desired.

2. Dilute the drug with cell culture medium to $2 x$ the final concentration with the highest final concentration at $10 \mu \mathrm{M}$ and serially dilute four times at a 1:10 ratio, for a total of five drug concentrations and a no drug control.

NOTE: If concentration of DMSO equals or exceeds $0.1 \% \mathrm{v} / \mathrm{v}$ at the highest drug dose, the no drug control should contain equivalent amount of DMSO to ensure the observed effects observed are not due to DMSO toxicity.

3. Pipette $100 \mu \mathrm{L}$ of drug solution into the appropriate well for a final drug concentration of $1 \mathrm{x}$ diluted in media.

\section{Image Acquisition}

NOTE: Images were acquired on days 0, 2 and 3. Depending on cell types and cellular processes being studied, other desired time points may be studied.

1. Stain cells to prepare for imaging.

1. Make up the dye solution at the following final concentrations

1. For fluorescence-based classification, prepare $5 \mu \mathrm{g} / \mathrm{mL}$ nuclear stain and $5 \mu \mathrm{M}$ dead cell stain in PBS (see Table of Materials). 
2. For morphology-based classification, prepare $5 \mu \mathrm{g} / \mathrm{mL}$ nuclear stain, $5 \mu \mathrm{M}$ dead cell stain, and $5 \mu \mathrm{M}$ cell stain in PBS (see Table of Materials).

2. Add $20 \mu \mathrm{L}$ dye solution to each well. Incubate for $30 \mathrm{~min}$ at $37^{\circ} \mathrm{C}$ protected from light.

\section{Optimize and acquire images.}

1. Remove the well plate from incubator, wipe the bottom of plate with $70 \% \mathrm{EtOH}$, and place the plate in the imaging chamber.

2. In the 'Setup' tab, click the '+' button under 'Channel Selection' to add appropriate channels to image (i.e. brightfield, nuclear stain, dead cell stain, GFP, and RFP signals).

3. Click 'Layout Selection', and take z-stacked test images every $2 \mu \mathrm{m}$ starting at $0 \mu \mathrm{m}$ and ending at $20 \mu \mathrm{m}$ to identify the plane of focus. Input this distance for every channel under 'Height'.

4. Click 'Snapshot' under each channel to evaluate intensities and optimize exposure times. Enter higher or lower values under 'Time' as required.

5. On right side of screen, highlight appropriate wells (to be imaged) on the plate schematic. In the wells schematic below, highlight the twenty-five fields to be imaged in a similar manner.

6. Under 'Run Experiment', identify the plate in 'Plate Name' on left tab, and click the 'Start' button (underneath) to run the image acquisition protocol with a 10X objective to generate gray-scale TIFF images in the aforementioned channels. NOTE: Step-by-step instructions for imaging protocol may differ between instruments, but parameter values should still be optimized.

7. Repeat the imaging on days two and three.

\section{Image Analysis}

NOTE: All image analysis was performed using proprietary software. However, because this is not a publicly available, comparable analyses were also designed on CellProfiler 2.2 and CellProfiler Analyst 2.0, with a brief protocol listed below and detailed protocol provided as supplementary material (test images are already loaded into pipelines to test workflow). The images from this experiment were grouped together on a per-well basis so that each well could be loaded individually. The CellProfiler pipelines below contain a regular-expression that parses metadata information from each image filename and allows the images to be further grouped by channel.

1. Download and install the open source software: 'CellProfiler' and 'CellProfiler Analyst'. Accept all default options and add-ons during installation.

2. Classify heterocellular cultures into subpopulations.

1. Open 'CellProfiler' software, click 'File'| 'Import Pipeline'| 'From File,' and select the appropriate file ("Fluorescence_Classification.cppipe" for fluorescence-based classification, or "Morphology_Classification.cppipe" for morphologybased classification).

NOTE: These files contain protocols for basic image processing and nuclei/cell segmentation. Because of the uneven Hoechst stain present across these cells, nuclei were segmented and enlarged, and then used to mask the image to allow for segmentation of nuclei with lower intensities.

1. Select the fluorescence-based classification pipeline to classify cells as either H3255 or CCD-19Lu based upon eGFP intensity, and to calculate morphology features.

NOTE: CCD-19Lu cells were transduced with GFP.

2. Select the morphology-based classification pipeline to segment nuclei and cells, and to calculate morphology features. NOTE: Further downstream analysis in 'CellProfiler Analyst' is needed for classification. Dead cells are classified via fluorescence-based classification.

2. Click 'View Output Settings' at the bottom left of the screen. Select the location of the image files for analysis ('Default Input Folder') and the destination for the extracted data ('Default Output Folder').

3. Click 'Analyze Images' to begin the analysis. When analysis is complete, click 'OK' and go to the 'Default Output Folder' to view the calculated data.

NOTE: Example parameter values and images are available in Supplemental File 1-CellProfilerProtocol.pdf.

4. For morphology-based classification (only) do the following.

1. Open 'CellProfiler Analyst' software, select the database properties file generated in CellProfiler, and click 'Open'.

2. Click the 'Classifier' tab at the top-left of screen. To call random cell images from experiment, click 'Fetch'; images will appear in the unclassified window.

5. Manually classify cells as positive (H3255) or negative (CCD-19Lu) by selecting and dragging cells to appropriate bin (see Supplemental File 2-Pipeline.pdf: Figure B). After classifying at least 50 cells per subpopulation, click 'Train Classifier', and then click 'Check Progress'.

NOTE: Cells are classified via a user-supervised random forest machine learning algorithm ${ }^{12}$. If the accuracy is not above approved threshold $(>90 \%)$, it may be necessary to go back and optimize cell segmentation on 'CellProfiler', or the cells may not be ideal for classifying by morphology.

6. Click 'Score All' to generate a table with cell counts for each subpopulation. 


\section{Representative Results}

We generated an image set consisting of 25 fields/well, 54 wells/plate ( 3 cell populations $\times 6$ drug concentrations $\times 3$ replicates), across three plates for a total of 4,050 individual images. The image sets generated over the course of the experiment were analyzed using proprietary software (see table of materials) to extract various quantitative properties of cells (i.e. morphology, fluorescence) which could then be used to classify cell subpopulations. However, because the commercial software used has limited access, comparable downstream pipelines in CellProfiler and CellProfiler Analyst were created.

\section{Heterocellular Classification into Subpopulations}

Nuclei were identified and segmented based on the DNA stain (here Hoechst) and cell populations were classified either based upon fluorescence or morphology (Figure 1). For fluorescence-based classification, the fibroblasts (CCD-19Lu) were previously transduced with GFPlentivirus. The GFP intensity levels were measured for each nucleus, and those that were calculated above the accepted threshold (based on the background signal) were classified as CCD-19Lu while those below were identified as tumor cells (H3255). For morphology-based classification, cells were previously stained with a non-toxic cellular stain (see the table of materials) and this was used to identify and segment the cytoplasm. A machine learning algorithm was trained with $\sim 50-100$ cells from each population. Morphological features were identified that were significantly different between the populations, which were then used to design a linear classifier to distinguish between CCD-19Lu and H3255 cells. The fluorescence and morphology classification protocols were $97.4 \%(n=1403)$ concordant at distinguishing between the two cell populations in untreated conditions and $92.5 \%(n=916)$ concordant in drug treated conditions $(1 \mu \mathrm{M}$ erlotinib) (Figure 2$)$.

\section{Phenotypic Analyses of Subpopulations}

In addition to discriminating between cell types, we aimed to characterize phenotypic properties of each subpopulation. Multiplexing assays saves time and reagents, adds consistency, and provides additional information regarding the system being studied. There are many potential phenotypic outputs and one should choose them based upon the questions of interest. Here, changes in the cell morphology and viability status in response to erlotinib treatment were investigated. After three days of drug treatment, a decrease in nuclear area and an increase in cellular area of the H3255 cells (Figure 3A) was observed. The mean difference in nuclear area between the "no drug" and "drug" treated populations was found to be statistically significant via a two-sided type-2 (equal variance) $t$-test $\left(p=7.92 \times 10^{-16}\right)$. We hypothesize that this observation is a cellular response to the stress imposed by drug treatment.

It is also of interest to study whether a drug has a cytotoxic (i.e. increase in number of dead cells over time) or cytostatic (i.e. decrease in number of cell births over time) effect on cells, as this has profound clinical impact. For example, a cytostatic drug effect induces growth arrest yet does not eliminate the cells from the tumor, thus there is the potential for cancer cells to reinitiate cell proliferation once the drug is removed. Drug effects can often be context, concentration, and cell type dependent. We previously observed erlotinib eliciting a cytotoxic response in one cell type, while showing a cytostatic response in another ${ }^{13}$.

Traditional viability assays output relative cell number and therefore, do not discriminate between growth arrests and cell death. Herein, dead cells were identified based upon propidium iodide stain (Figure 3B). Both cytotoxic and cytostatic effects of erlotinib on $\mathrm{H} 3255$ cells were observed, with an increase in the number of deaths and a decrease in the number of births following drug treatment (Figure $\mathbf{3 C}$ ). It is worth noting that the number of dead cells drops following day 1 likely due to cell clearance. CCD-19Lu cells were not affected by the drug. An additional advantage of this platform is the generation of quantitative data. For example, in our co-culture experiment, an initial subpopulation of $1,118(75.8 \%) \mathrm{H} 3255$ cells was found to be $2,817(87.9 \%)$ or $396(57.2 \%)$ after three days without or with erlotinib treatment, respectively (Figure 4). Because we can generate actual cell counts instead of relative percentage (as with flow cytometry methods), we conclude that the change in composition during drug treatment is due to a decrease in H3255 cells and not an increase in CCD-19Lu. It is worth nothing that death rates may be underestimated due to cell clearance, which is difficult to assess experimentally and likely differs across cell types. 


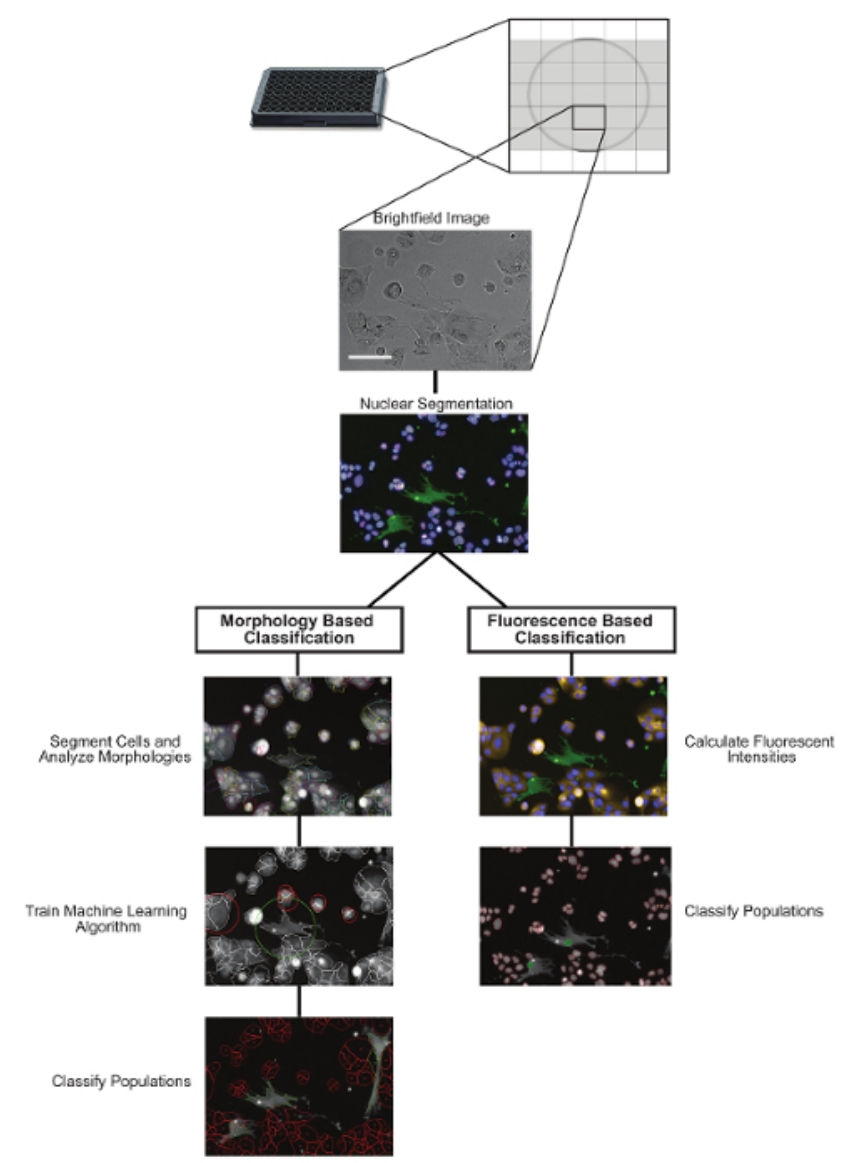

Figure 1: Overview of the Image Analysis Protocol. Two potential downstream image analysis pipelines to classify heterocellular populations using either morphology-based classification or fluorescence-based classification. Scale bars $=100 \mu \mathrm{m}$. Please click here to view a larger version of this figure. 
A

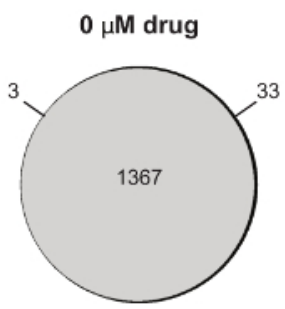

$\square$ Morphology $\square$ Morphology + Fluorescence

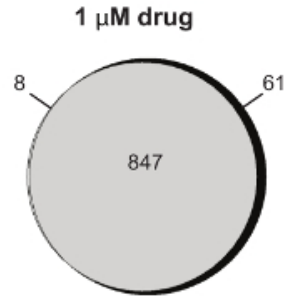

Fluorescence

B

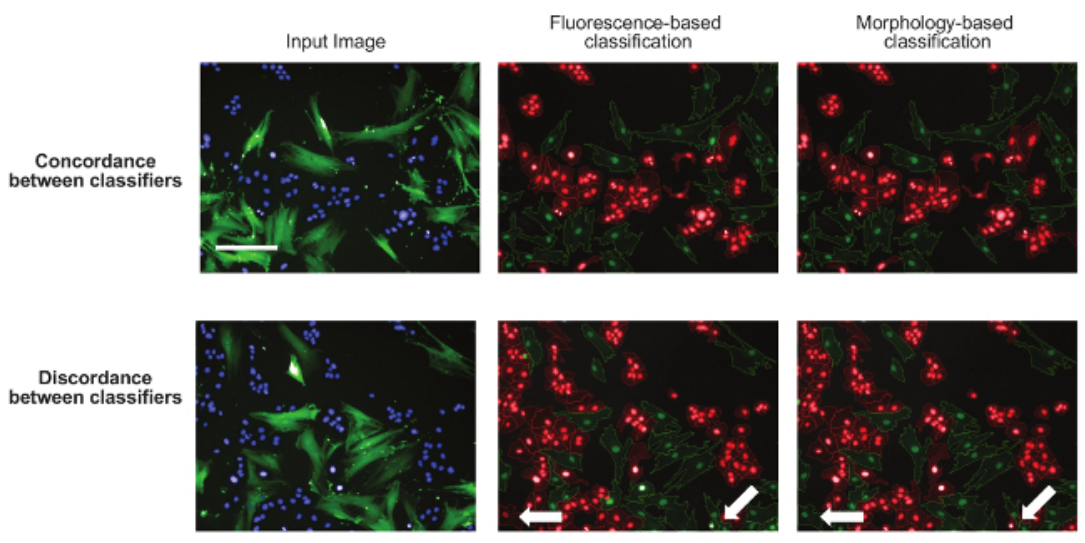

Figure 2: Concordance between Morphology and Fluorescence-based Classification. (A) Concordance plot displaying the overlap of the two classification protocols. The same cells were classified as H3255 using both morphology-and fluorescence-based classification. The two protocols were in agreement, with classification for $97.4 \%(n=1403)$ of untreated cells and $92.5 \%(n=916)$ of cells treated with erlotinib (Note: white area is too small to visualize). (B) 10X images depicting examples of good and poor concordance between fluorescence-based and morphology-based classification. The white arrows point out cells that were inconsistently classified between platforms. Input image: blue nuclei (Hoechst); green - CCD19Lu (GFP). Classification images: Red - H3255; green - CCD-19Lu. Scale bar $=100 \mu \mathrm{m}$. Please click here to view a larger version of this figure. 
A
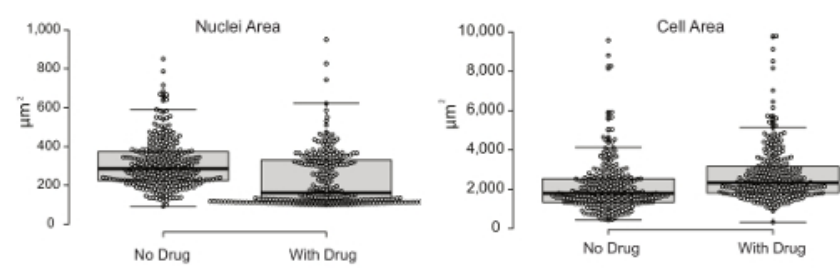

B
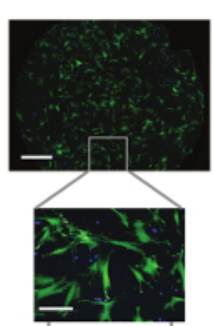

C
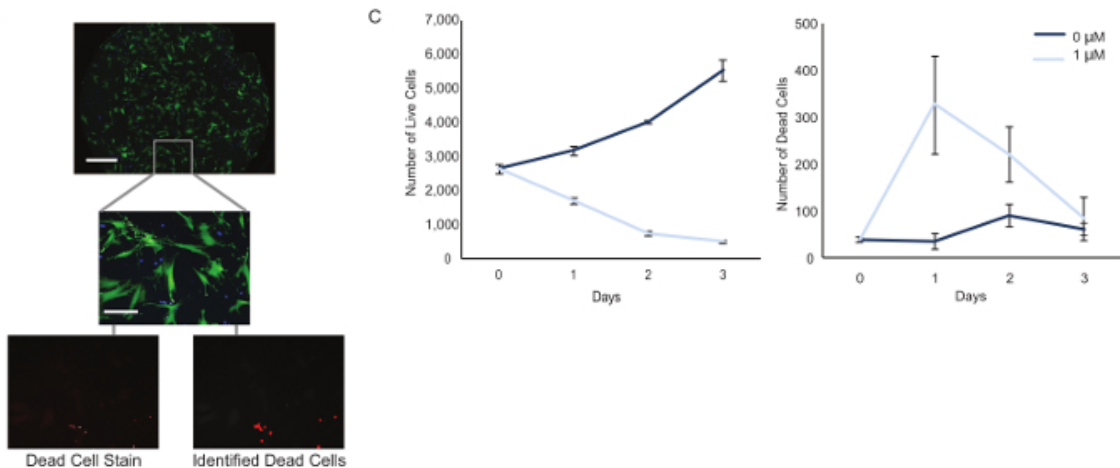

Figure 3: Multiplexed Phenotypic Measurements from a Single Experimental Setup. (A) Morphological features, such as nuclei and cell area, were calculated on the single cell level in the presence and absence of drug. Note: Cell areas measuring smaller than $100 \mu \mathrm{m}^{2}$ were considered debris and excluded from analyses. Box plot depicts median with first and third quartile ranges and $95 \%$ confidence interval error bars. (B) H3255 (blue) and CCD-19Lu (green) cells were co-cultured and dead cells were identified based upon the intensity of propidium iodide stain (red) and imaged using a 10X objective. Scale bar = $1 \mathrm{~mm}$ (top panel); $100 \mu \mathrm{m}$ (bottom images). (C) Total number of live and dead cells were calculated over three days with or without drug treatment, with an obvious decrease in number of live cells and increase in dead cells with the addition of erlotinib. Error bars represent standard error of the mean based on of three replicates. Please click here to view a larger version of this figure. 
Day 0
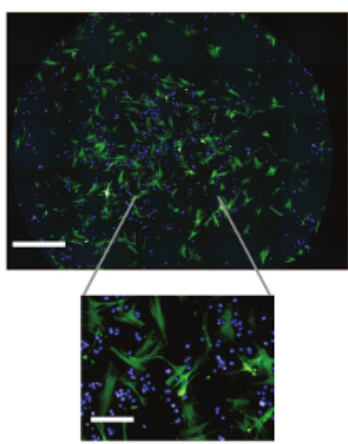

Day 3

$0 \mu \mathrm{M}$ drug
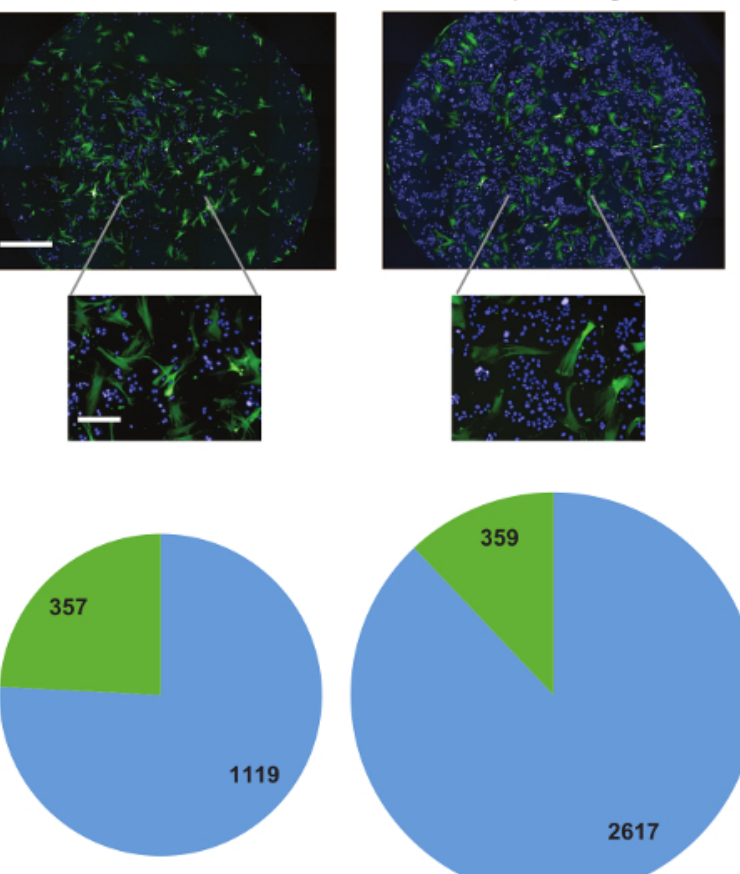

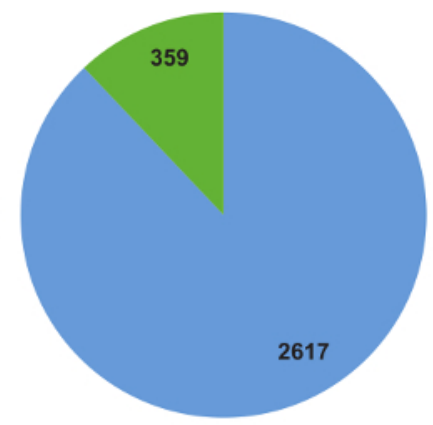

- CCD-19Lu

- H3255
Day 3

$1 \mu \mathrm{M}$ drug
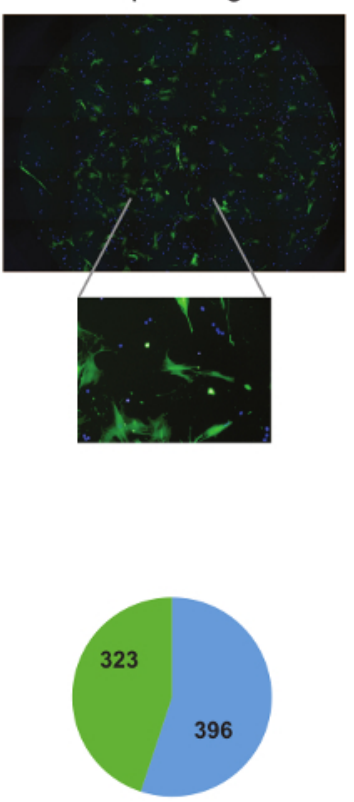

Figure 4: Subpopulation Dynamics over Time. Representative 10X images of wells containing H3255 (blue) and CCD-19Lu (green) on day 0 or day 3 with and without drug. Cells belonging to each subpopulation were counted and proportional pie charts show the actual change in population composition across samples. Scale bars $=1 \mathrm{~mm}$ (middle panels, shpwn in ;eftmost panel), $1 \mathrm{~mm}$ (top image), $100 \mu \mathrm{m}$ (bottom images). Please click here to view a larger version of this figure.

\section{Discussion}

The protocol described above improves upon current cell biology assays by providing more comprehensive insights into phenotypic dynamics of multiple cell types in response to environmental perturbations while using reduced reagents and time. A major advantage of this experimental design is the ability to analyze multiple phenotypes with a single setup and generate quantitative data characterizing these phenotypes on a single cell level. One technical advantage to this platform is the ease of initial troubleshooting compared to other assays. Because this method is image-based, one is able to visualize the wells for apparent over/under seeding. It is advisable to have cells in the exponential growth phase for the duration of the experiment and not be limited by nutrient or spatial constraints or confounded by senescence due to scarce seeding. Otherwise, birth and death rates may not be reproducible between experiments. For reference, CellPD is a publicly available program for computation of birth and death rates ${ }^{14}$. Additionally, one can visualize whether adequate concentrations of dyes were added to each well. Pipetting issues in an individual well could result in missegmentation and skewed data, but can easily be detected with the aforementioned protocol.

Unfortunately, not all cell types may be amenable to this application. It is important to be able to accurately segment the nuclei and cells, therefore analysis of cells that organize in more sphere-like or clumped structures may not be suitable. For some cells, it also may be advantageous to use a cell strainer prior to seeding to ensure initial seeding of single cells. In addition, the linear classifier technique is only applicable to cell types that can be readily distinguished based on morphology features.

For the success of the protocol, it is important to first optimize the imaging conditions, as the validity of downstream analyses is dependent on the quality of the images. While here we performed experiments using a high-content screening platform, image acquisition can also be performed using any fluorescent microscope (although an automated imaging platform is ideal for high-throughput approaches). Test images should be taken prior to each imaging time point to ensure that there are no problems with the microscope or protocol. The signal to noise ratio should be high, especially for the channels that will be used for segmentation (i.e. nuclei, cell stains). Additionally, it is important to image in the optimal plane of focus. If the images are out of focus, segmentation becomes much more difficult and the calculated morphological features will likely be inaccurate. Illumination differences between fields can cause problems with image segmentation as well. Large differences in brightness make the automated selection of threshold values difficult. Additionally, if there are heterogeneous fluorescence intensities between cells, a single threshold value may not sufficiently segment all the cells in an image. In this analysis, these problems were overcome by creating masks around brighter and dimmer cell populations and segmenting each population separately. 
While the phenotypes under investigation in this protocol are limited to live, dead, and morphological characterization, they can easily be expanded to investigate other features. For example, functional genetic studies can be added with RNAi, overexpression, or other chemical perturbations.

In this paper, the capabilities of the protocol to measure the response of non-small cell lung cancer cells to erlotinib in the presence and absence of CAFs was demonstrated. However, this is merely one example of the many cell types and microenvironmental parameters that can be tested. We have extended this protocol to be used with other cell types and drug studies, including primary cells isolated from patient tumors ${ }^{13,15}$.

\section{Disclosures}

The authors have nothing to disclose.

\section{Acknowledgements}

This work was funded by National Cancer Institute (NCI) Grants U54CA143798 and U54CA143907 to establish Physical Sciences-Oncology Centers (PS-OCs) at the Dana-Farber Cancer Institute and University of Southern California, respectively. S.M. Mumenthaler received a PS-OC transnetwork award that supported some of this work.

We would like to express our deepest gratitude to our philanthropic supporters, particularly the Stephenson family, Emmet, Toni and Tessa, for their donation of the Operetta HCS platform. We would also like to thank J. Foo for guidance, and the Center for Applied Molecular Medicine team members: D. Agus for clinical guidance and mentorship, K. Patsch for meaningful discussions with experimental design, R. Rawat for aid in image analysis protocols, J. Katz for technical assistance with the Operetta, and P. Macklin and D. Ruderman for helpful discussions and feedback.

\section{References}

1. Kanemura, Y. et al. Evaluation of in vitro proliferative activity of human fetal neural stem/progenitor cells using indirect measurements of viable cells based on cellular metabolic activity. J Neurosci Res. 69 (6), 869-879 (2002).

2. Plumb, J. A., Milroy, R., \& Kaye, S. B. Effects of the pH dependence of 3-(4,5-dimethylthiazol-2-yl)-2,5-diphenyl-tetrazolium bromideformazan absorption on chemosensitivity determined by a novel tetrazolium-based assay. Cancer Res. 49 (16), $4435-4440$ (1989).

3. Hsu, S. et al. Green tea polyphenols induce differentiation and proliferation in epidermal keratinocytes. J Pharmacol Exp Ther. 306 (1), 29-34 (2003).

4. Caicedo, J. C., Singh, S., \& Carpenter, A. E. Applications in image-based profiling of perturbations. Curr Opin Biotechnol. 39 134-142 (2016).

5. Sero, J. E. et al. Cell shape and the microenvironment regulate nuclear translocation of NF-kappaB in breast epithelial and tumor cells. Mol Syst Biol. 11 (3), 790 (2015).

6. Chen, J. F. et al. Subclassification of prostate cancer circulating tumor cells by nuclear size reveals very small nuclear circulating tumor cells in patients with visceral metastases. Cancer. 121 (18), 3240-3251 (2015).

7. Ohlund, D., Elyada, E., \& Tuveson, D. Fibroblast heterogeneity in the cancer wound. J Exp Med. 211 (8), 1503-1523 (2014).

8. Paraiso, K. H., \& Smalley, K. S. Fibroblast-mediated drug resistance in cancer. Biochem Pharmacol. 85 (8), 1033-1041 (2013).

9. Kalluri, R. The biology and function of fibroblasts in cancer. Nat Rev Cancer. 16 (9), 582-598 (2016).

10. Carpenter, A. E. et al. CellProfiler: image analysis software for identifying and quantifying cell phenotypes. Genome Biol. 7 (10), R100 (2006).

11. Jones, T. R. et al. CellProfiler Analyst: data exploration and analysis software for complex image-based screens. BMC Bioinformatics. 9482 (2008).

12. Dao, D. et al. CellProfiler Analyst: interactive data exploration, analysis and classification of large biological image sets. Bioinformatics. 32 (20), 3210-3212 (2016).

13. Garvey, C. M. et al. A high-content image-based method for quantitatively studying context-dependent cell population dynamics. Sci Rep. 6 29752 (2016)

14. Juarez, E. F. et al. Quantifying differences in cell line population dynamics using CellPD. BMC Syst Biol. 10 (1), 92 (2016).

15. Mumenthaler, S. M. et al. The Impact of Microenvironmental Heterogeneity on the Evolution of Drug Resistance in Cancer Cells. Cancer Inform. 14 (Suppl 4), 19-31 (2015). 